\title{
WAKE VORTEX TRANSPORT IN PROXIMITY TO THE GROUND
}

\author{
David W. Hamilton \\ and \\ Fred H. Proctor \\ NASA Langley Research Center \\ Mail Stop 156A \\ Hampton, Virginia 23681-2199
}

$19^{\text {th }}$ Digital Avionics Systems Conference

7-13 October 2000, Philadelphia, Pennsylvania, AIAA and IEEE 


\title{
WAKE VOR'TEX TRANSPOR'T IN PROXIMITY TO THE GROUND
}

\author{
David W. Hamilton' and Fred Proctor ${ }^{2}$ \\ NASA Langley Research Center, Hampton, Virginia
}

\begin{abstract}
A sensitivity study for aircraft wake vortex transport has been conducted using a validated large eddy simulation (LES) model. The study assumes neutrally stratified and nonturbulent environments and includes the consequences of the ground. The numerical results show that the nondimensional lateral transport is primarily influenced by the magnitude of the ambient crosswind and is insensitive to aircraft type. In most of the simulations, the ground effect extends the lateral position of the downwind vortex about one initial vortex spacing $\left(b_{0}\right)$ in the downstream direction. Further extension by as much as one $b_{0}$ occurs when the downwind vortex remains "in ground effect" (IGE) for relatively long periods of time. Results also show that a layer-averaged ambient wind velocity can be used to bound the time for lateral transport of wake vortices to insure safe operations on a parallel runway.
\end{abstract}

\section{Nomenclature}

$b_{0} \quad$ initial vortex separation

$L \quad$ lateral distance traveled by the downwind vortex

$T \quad$ nondimensional time; $t V_{o} / b_{0}$

$t$ time

$\Delta t \quad$ time step

$U \quad$ crosswind component of velocity

$U_{\text {max }}$ ambient crosswind velocity at elevations $z_{\text {top }}$ and above

$\bar{U}_{e} \quad$ layer-averaged ambient crosswind velocity

$U_{\text {. }} \quad$ ambient crosswind velocity

$V_{1} \quad$ initial vortex descent velocity; $\Gamma_{0} /\left(2 \pi b_{0}\right)$

$y_{6} \quad$ initial vortex lateral position

$y_{i r i f t} \quad$ drift model lateral position

$y_{\text {irass }}$ TASS vortex lateral position

$x, y, z$ longitudinal, lateral, vertical space coordinate

$z_{i} \quad$ altitude of initial vortex

\footnotetext{
${ }^{1}$ Research Scientist, Airborne Systems Compelency

${ }^{2}$ Research Scientist, Airborne Systems Competency, AI A $A$ member
}

$\begin{array}{ll}z_{\text {sop }} & \begin{array}{l}\text { altitude above which the ambient wind } \\ \text { velocity becomes constant }\end{array} \\ z_{w} & \text { altitude of vortex core } \\ u & \text { power function in ambient wind profiles } \\ \Gamma_{0} & \text { initial vortex circulation }\end{array}$

\section{Introduction}

The National Aeronautics and Space Administration is making efforts through the Terminal Area Productivity Program to safely increase terminal capacity and reduce delays to meet the increasing air travel demands. Particularly, the Aircraft VOrtex Spacing System (AVOSS) integrates systems that assess the present and short-term weather state and make transport and decay predictions for aircraft wake vortices [1. $2,3]$. These predictions aim to safely decrease spacing between arriving and departing aircraft under instrument meteorological conditions (IMC) and effectively increase airport capacity. The initial goal of AVOSS is to successfully demonstrate a capability to increase throughput on a single runway. Currently, capacity is also reduced during IMC when operations on a parallel runway are either shutdown or reduced due to the uncertainties in the positions of laterally shifting wakes.

Presently, independent approaches with lateral separations of less that $2500 \mathrm{ft}(\sim 760 \mathrm{~m})$ are not allowed during IMC. This restriction is due to the danger of potential encounters with drifting vortices. Recent research has been directed at improving this limitation without jeopardizing safety $[4,5,6,7]$. Schilling [4] concluded that a minimum crosswind magnitude of $2.5 \mathrm{mss}^{-1}$ was necessary to transport vortices to the parallel runway ( $518 \mathrm{~m}$ separation) at the Frankfurt International Airport (FIA). This conclusion was partly based on numerical simulations and assumed a wake vortex lifetime of $180 \mathrm{~s}$. Kopp [5] used a continuous-wave lidar at FIA and found that a 
crosswind of $4 m s^{l}$ would transport a wake into the parallel runway safety area in less than $90 . s$. Rudis et al. [6] have observed wakes traveling beyond 500 $m$. in the presence of strong crosswinds and low turbulence. A model that incorporates factors such as mean crosswind. runway separation. and wake vortex lifetime is needed to insure safe operations on parallel runways.

A wake vortex is considered "in ground effect" (IGE) when its altitude is within one initial vortex separation $\left(h_{0}\right)$ above the ground. According to Critchley and Foot [8], most aircraft wake encounters occur between 30-60 m (100-200 ft). which for many aircraft is IGE. Wake vortex behavior IGE has been investigated both observationally $[5,6,9,10,11,12]$ and numerically $[4,7,13,14,15,16,17]$, but most of the research to date has only qualitatively described the complexities of this phenomenon. From recent large eddy simulation (LES) results, Proctor et al. [17] has proposed an empirical model quantifying the lateral spreading of a vortex pair once the vortex has reached its minimum height in ground effect. Their results were limited to cases with little or no ambient wind shear.

Vertical gradients in the ambient crosswind have been shown to affect wake vortex descent and lateral transport $[18,19,20]$. Specifically, nonlinear shear in the crosswind can reduce the descent rate, stall, and potentially lead to the rising of a wake vortex. The orientation of the wind shear gradient (i.e. $\sigma^{2} U / \% z^{2}$ positive or negative) will determine whether the upwind or the downwind vortex is more affected [19]. The combination of wind shear and ground effect, which often coincide, complicate the problem of predicting wake vortex transport.

The present study will investigate wake vortex transport in proximity of the ground in order to determine safe bounds for arriving and departing aircraft on a downwind parallel runway. The simulations conducted in this study assume environments with no ambient turbulence. It should be noted that turbulence influences vortex decay and can have an affect on transport [21]. For example, Han et al [22] have examined the influence of convective boundary layer turbulence eddies on wake vortex transport. They determined that substantial lateral shifting of the vortex can occur under the intluence of turbulence eddies. potentially masking the contributions of crosswind shear and ground effect. In the present study, turbulence effects will be ignored in order to isolate the ground effect but should be considered for future study.

In this study we subject a range of aircraft to varying ambient crosswind conditions. We will use these results to quantify the extent of IGE and present a bound on vortex transport to ensure safe operations on a downwind parallel runway.

\section{The Numerical Model and Initial Conditions}

The model employed in this study, the Terminal Area Simulation System (TASS) [23], is a compressible, LES model, which has successfully demonstrated wake vortex behavior in various meteorological conditions [16. 17, 19, 22, 24, 25. 26. 27, 28, 29, 30].

\section{Grid Configuration}

For the purpose of examining wake transport. and to save on computational time. the model is integrated in time using a two-dimensional domain. The two-dimensional domain inhibits vortex decay and prevents three-dimensional instabilities, such as Crow linking. from developing [31]. Given these limitations, previous studies have shown that simulations with the two-dimensional assumption have given valid results for vortex transport $[16,19$. 24, 30].

The coordinate system is chosen such that $x$ is along the flight path or longitudinal direction. $y$ is in the cross track or lateral direction and $z$ is height above ground. The experiments assume a uniform one meter grid size. The domain depth is designed so that two vortex separations $\left(2 b_{o}\right)$ exist between the initial vortex altitude $\left(z_{i}\right)$ and the top of the domain. The numerical grid translates with the downwind wake vortex: therefore, allowing the domain width to be independent of crosswind magnitude. In all experiments, the width of the domain is fixed at $11.5 \mathrm{bo}$. 


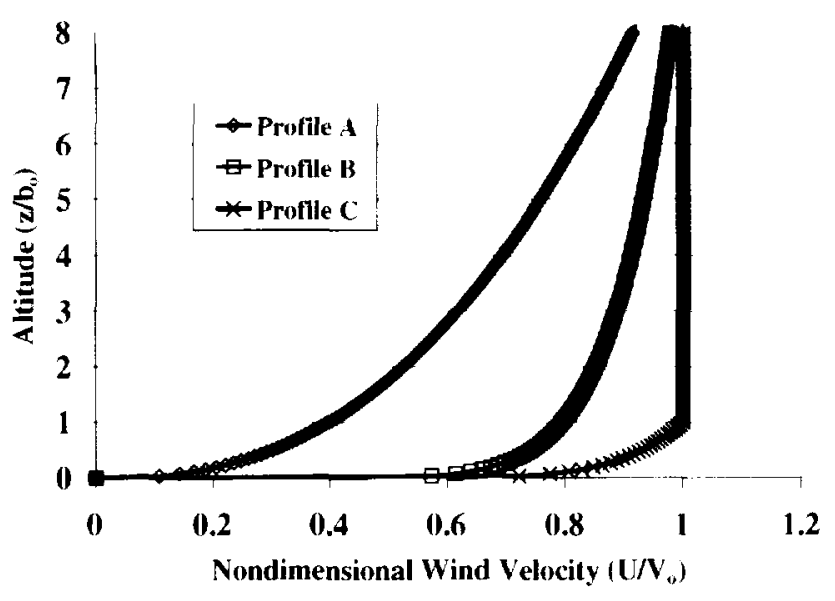

Figure 1: Ambient crosswind profiles for TASS simulations.

\section{Atmospheric Conditions}

The simulations are initialized with a neutrally stratified and dry atmosphere. The ambient crosswind is assumed to be horizontally uniform with its vertical distribution prescribed by the following power law:

$$
\begin{array}{ll}
U_{\rho}(z)=U_{\max }\left(\frac{z}{z_{t o p}}\right)^{\alpha} & \text { for } z<z_{(i p)} \\
U_{\rho}(z)=U_{\max } & \text { for } z \geq z_{i o p}
\end{array}
$$

Three wind profiles with contrasting intensities of shear are chosen for the sensitivity study (see Fig. 1). Four wind magnitudes are used in each profile and are scaled by the initial vortex descent rate of the aircraft tested, i.e. $U_{\max } / V_{0}=1,2,4$, and 8. For a typical Boeing 727 , these values correspond to a dimensional crosswind velocity of $U_{\max }=1.53,3.06,6.12$, and $12.24 \mathrm{~ms}^{-1}$. respectively $[1 \mathrm{~ms}=2 \mathrm{kts}]$. The parameters for the wind profiles are listed in Table 1.

Table 1. Values for wind profile parameters.

\begin{tabular}{cll}
\hline Profile & $a$ & $z_{\text {top }}$ \\
\hline A & 0.4 & $10 b_{0}$ \\
B & 0.1 & $10 b_{0}$ \\
C & 0.1 & $b_{0}$ \\
\hline
\end{tabular}

(a)
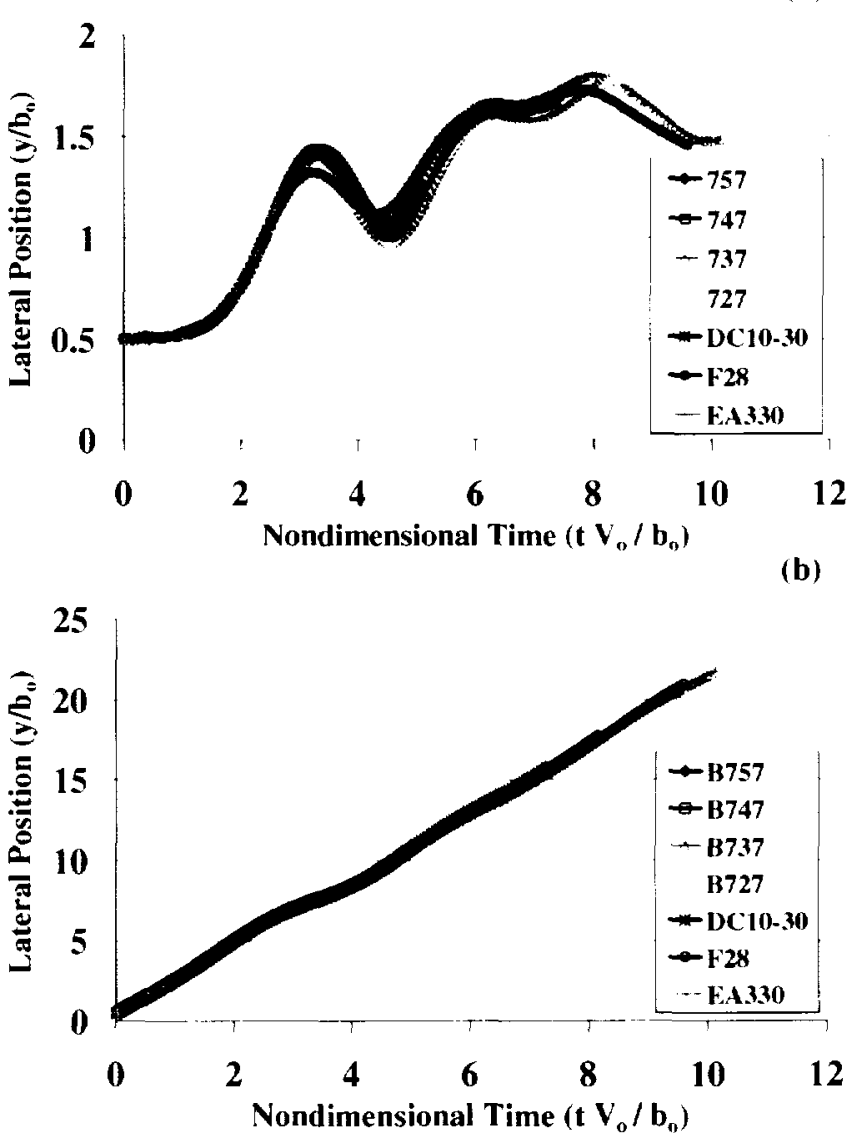

Figure 2: Sensitivity to aircraft type for TASS simulated trajectories for (a) calm wind and (b) crosswind cases. Figure $2 \mathrm{~b}$ is with wind profile "A", $U_{\max }=2 V_{o}$ and $z_{i}=1.5 b_{o}$.

\section{Vortex Initialization}

The initial vortex profile is representative of a post rollup. wake vortex velocity field. The vortex initialization is the same as that described in Proctor et al. [17]. The initial vortex parameters for the seven aircraft are listed in Table 2 and represent the three aircraft weight classes, i.e. small, large, and heavy. Six vortex initialization heights are tested: $z_{i} / h_{n}=0.5,1,1.5,2,4$, and 8 . The six cases were chosen to represent vortices initialized in. near. and out of ground effect [32]. 
Table 2. Initial vortex parameters.

\begin{tabular}{llll}
\hline Aircraft & $\boldsymbol{b}_{\boldsymbol{o}}(\boldsymbol{m})$ & $\boldsymbol{V}_{\boldsymbol{o}}\left(\boldsymbol{m} \boldsymbol{s}^{-1}\right)$ & $\boldsymbol{\Gamma}_{\mathrm{o}}\left(\boldsymbol{m}^{2} \boldsymbol{s}^{-1}\right)$ \\
727 & 26 & 1.53 & 250 \\
737 & 22 & 1.49 & 205 \\
747 & 50 & 1.70 & 534 \\
757 & 30 & 1.63 & 307 \\
F28 & 20 & 1.28 & 161 \\
EA 330 & 47 & 1.28 & 378 \\
DC $10-30$ & 40 & 1.96 & 493 \\
\hline
\end{tabular}

\section{Results}

\section{Sensitivity to Different Aircraft}

To determine if wake vortex transport is sensitive to the generating aircraft, two sets of experiments are conducted using the seven aircraft listed in Table 2. Only the downwind (downshear) vortex is analyzed since it is usually the first to impact parallel runway operations.

The first experiment examines the lateral transport of the downwind vortex in an atmosphere without crosswind (calm wind). A comparison of the simulations using conventional nondimensionalizations [33] is shown in Fig. 2a. During the initial period of the simulation $(T<1)$ the vortices descend due to their mutual induction and exhibit no lateral displacement. By $T=2$, the vortices lose their vertical motion and spread laterally due to ground effect [34]. Between $T=3$ and 5, viscous interaction with the ground causes the development of a shear layer with opposite sign vorticity to that of the primary vortices. This shear layer separates from the surface and is transported around the periphery of each of the vortices [9]. Secondary vortices are generated within this shear layer and orbit the primary vortices. The circulation from the secondary vortices causes the primary vortices to rise and bounce. Later, the primary vortices begin to oscillate under the influence of the orbiting secondary vortices. It should be noted that the 2-D simulations might exaggerate these oscillations [16]. However, comparisons with observed vortex behavior have shown good predictions by the 2-D simulations $[16,24,30]$.

The second experiment examines lateral transport in an atmosphere with crosswind (Fig. 2b). Similar to the calm wind cases, the curves for lateral transport collapse when nondimensionalized. revealing the insensitivity to aircraft type. The effects of the ground and secondary vortices on vortex motion (Fig. 2a) appear to be less significant due to the dominating influence of advection. i.e. the process of transport by the crosswind velocity field. In fact. by $T=8$, the lateral position of the vortices is an order of magnitude greater than of those in calm wind. thus revealing the dominance of advection

\section{A "Drift Model" Analysis}

An additional 72 simulations have been conducted examining wake vortex transport near the ground in a variety of crosswind conditions. The simulations involve a mix of the three wind profiles (Fig. 1), four wind magnitudes $\left(U_{m a x}\right)$, and six initial vortex altitudes $\left(z_{i}\right)$. which were defined earlier. A simple relationship for vortex advection. i.e. a drift model, has been calculated for each simulation:

$$
y_{d r i j i}(t)=y_{d r i f i}(t-\Delta t)+U_{e}\left(z_{w}\right) \Delta t
$$

where $U_{e}$ is from Eq. (1). In this calculation, $z_{w}$. the height of the vortex at time $t$, is taken from the TASS simulation.

In order to evaluate the effects of ground and the nonlinear interaction with a non-uniform crosswind profile. the difference is taken between Eq. 2 and the lateral position predicted by the TASS simulation. The resulting 'drift differences' show that in nearly all cases. TASS predicts a lateral position downwind of the position calculated by the drift model (Fig. 3). This downwind extension indicates that transport of the downwind vortex IGE is greater than that due to advection alone. The majority of the 'drift difference' curves asymptote close to one $b_{0}$ (see Fig. 3). Thus, the ground effect extends the lateral position of the downwind vortex to about one initial vortex spacing.

A few cases exhibit a 'drift difference' (extension) greater than one $b_{o}$. This further extension is attributed to either multiple IGE encounters or a prolonged ground interaction. Figure 4 shows a vortex path revealing a prolonged ground interaction. The vortex initially sinks and quickly rebounds to a height of $1.5 b_{,}$. then it begins to oscillate near $z=b_{0}$. Lidar measurements from the Memphis-95 field study provided observational 
(a)

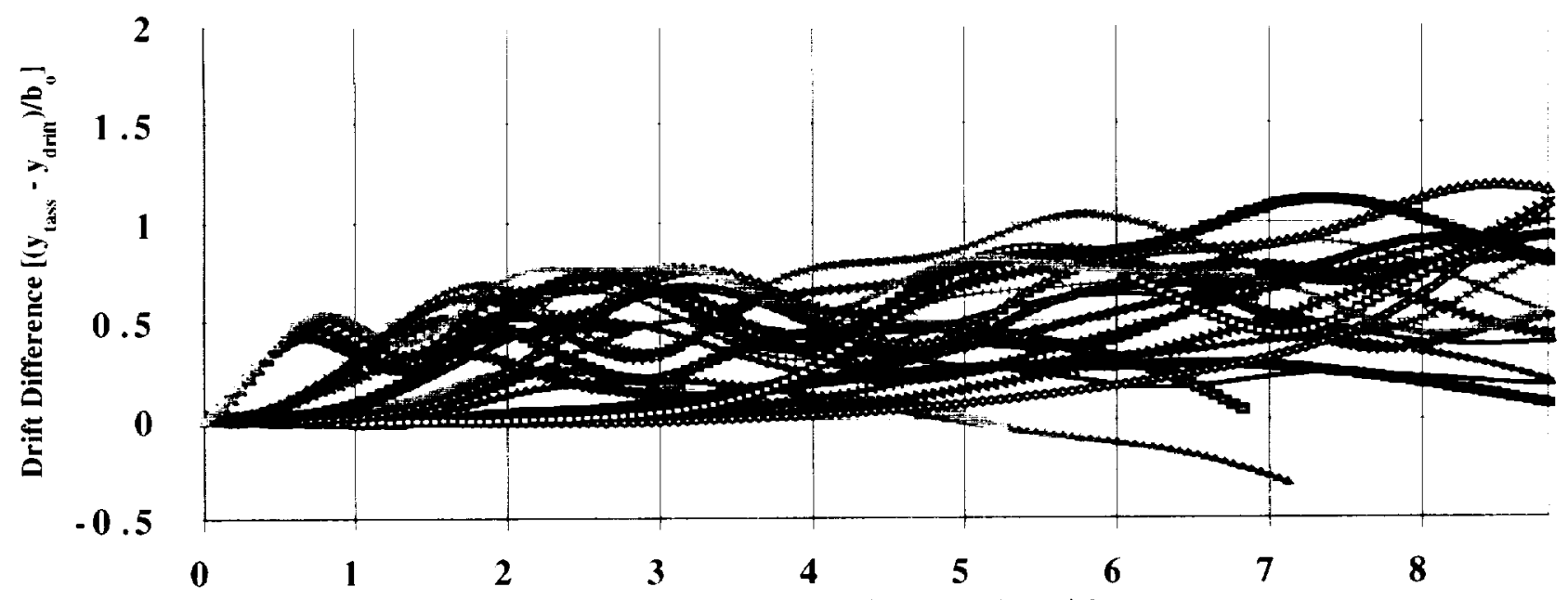

Nondimensional Time ( $\mathrm{T}$ o/ bo)

(b)
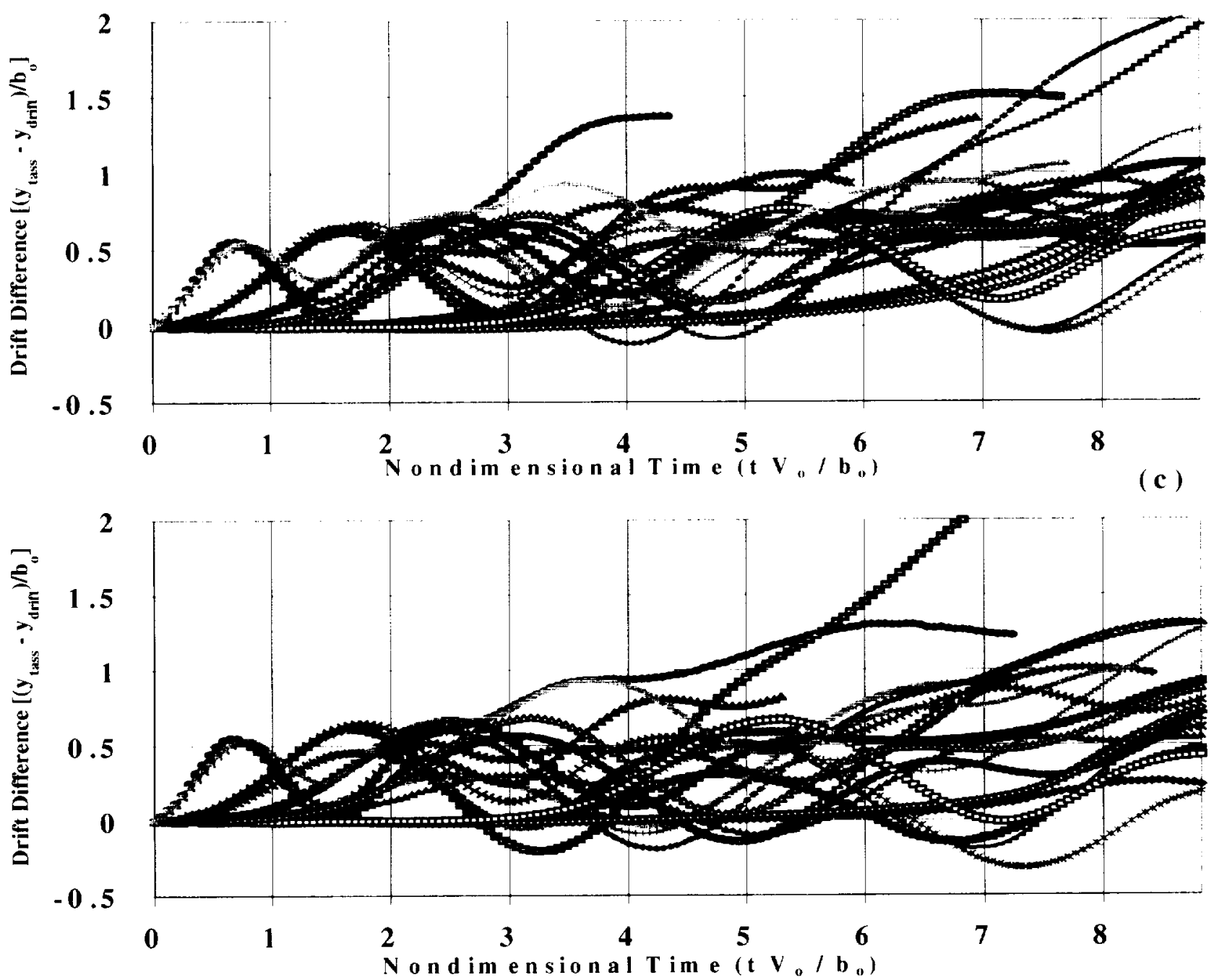

Figure 3: Drift differences between TASS prediction and drift model (Eq. 2) for cases with (a) wind profile "A", (b) wind profile "B", and (c) wind profile "C". 
evidence of similar wake paths [35]. Of 80 IGF cases identified, $8(10 \%)$ displayed a prolonged ground interaction that is similar to that depicted in Fig. 4.

It appears that the prolonged ground interaction can be related to the ambient wind shear and the initial vortex altitude. For simulations with relatively small magnitudes of ambient crosswind shear gradient, i.e. $\left|\hat{\sigma}^{2} U / \hat{\mathrm{s}} \mathrm{z}^{-2}\right|<0.001 \mathrm{~m}^{-1} \mathrm{~s}^{-1}$. there was little affect on the vortex descent rate. In these cases, a prolonged ground interaction occurred and drift differences were greater than one $b_{0}$. On the other hand. for environments with relatively large magnitudes of the crosswind shear gradient. i.e. $\left|r^{2} U / C z^{2}\right| \geq 0.001 m^{-1} s^{-l}$. the descent rate was reduced. For these cases the vortices ascended after ground interaction, and their drift differences remained bounded by one $b_{s,}$. The vortex initialization height can be as important as the magnitude of shear. When the vortex was initialized IGE, i.e. $z_{i}=0.5 b_{i}$, a prolonged ground interaction occurred regardless of the magnitude of the ambient wind shear gradient. In these simulations, the vortex eventually ascended above $z=b_{o}$, and the drift difference exceeded one $b_{0}$.

The drift model analysis shows that the ground effect extends the lateral position of the downwind vortex to about one initial vortex spacing $\left(b_{i}\right)$, relative to transport without ground effect. Further extension beyond one $b_{0}$ occurs when the downwind vortex either remains in ground effect for long periods of time or rebounds in ground effect more than once.



Figure 4: Vortex trajectory for the TASS case with wind profile "A", $U_{\max }=4 V_{o}$, and $z_{i}=1 b_{o}$.

\section{A "Time-to-Cross" Analysis}

A drawback from implementing the results of the previous section into an operational system is knowledge of the vortex altitude. A layer-averaged approach is defined and evaluated for a prediction of vortex lateral transport. rather than estimating the height of the vortex. This approach considers neither ground effect nor the vertical trajectory of the vortex. and is used to predict the transport of the downwind vortex.

The time interval for the downwind vortex to travel across a selected distance is compared between the layer-averaged prediction and the TASS simulation (Fig. 5). The layer-average prediction is determined by $t=L / \bar{U}$, where $\bar{U}$ is the layer-averaged ambient crosswind velocity (averaged between $z=0.5 b_{i}$, and $z=z_{i}+0.5 b_{0}$ ).

$$
\bar{U}=\frac{\int_{0.5 b_{n}}^{z_{1}+0.5 b_{n}} U_{p} d z}{\int_{0.5 b_{o}}^{z_{i}+0.5 b_{n}} z d z}
$$

and where $L$ is an arbitrary distance from the initial vortex position. The predictions are determined for values of $L$ that range from 100 to $800 \mathrm{~m}$ by $100 \mathrm{~m}$ increments.

An empirical relationship for bounding the time interval for lateral transport of the downwind vortex IGF is determined from the data in Fig. 5. and is:

$$
t=0.688 L \bar{U}
$$

The above relationship (i.e. transport bound. Fig. 5) represents the minimum time interval for the vortex to transport across distance $L$ ( $L$ can represent the separation between parallel runways). The thin line in Fig. 5 represents $t=L / \bar{U}$. which is the transport by simple advection.

This analysis is applied to nine observed wake vortices and is shown also in Fig. 5. The observed wake positions were measured by lidar during the Memphis-95 field experiment [35]. The wind profiles used in this analysis were from measurements near the lidar site. The predicted values for the observed vortices are within or just outside of the transport bound. 


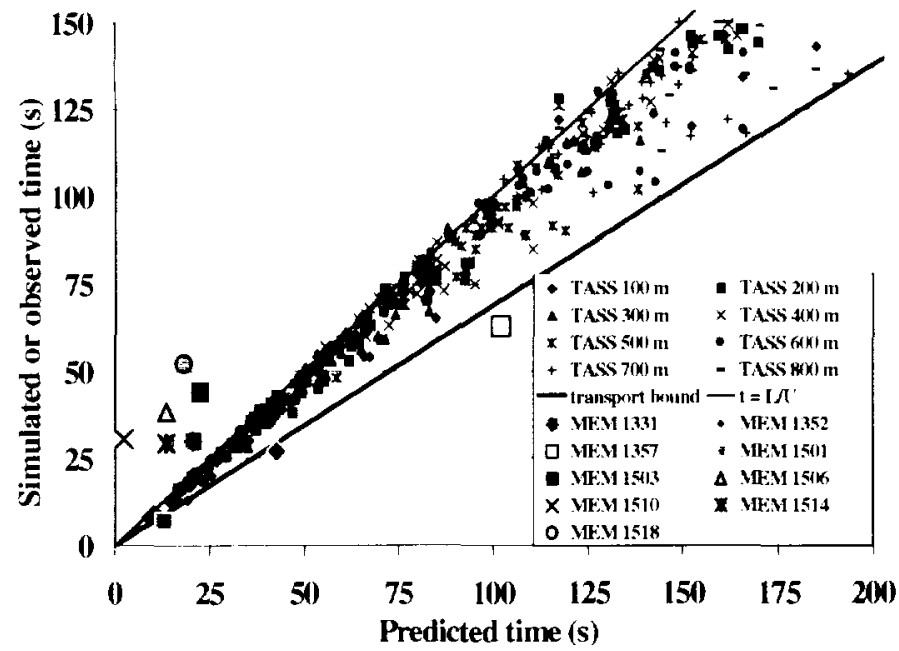

Figure 5: Time to travel distance $L$ from prediction with Eq. 3 versus TASS simulation (small symbols). Also included is the prediction versus observed time for several Memphis cases (large symbols).

\section{Summary and Conclusions}

Wake vortex transport in the proximity of the ground has been examined. The simulations conducted assume 1) no ambient turbulence. 2) little to no vortex decay, 3) mean crosswind profiles, and 4) neutral stratification. Results of the simulations are used to evaluate lateral transport bounds in ground effect. The conclusions of the present study are:

- Lateral transport is primarily influenced by the magnitude of the ambient crosswind.

- Nondimensional lateral transport is insensitive to aircraft type.

- The ground effect extends the lateral position of the downwind vortex to about one initial vortex spacing $\left(b_{0}\right)$, relative to transport without ground effect.

- Further extension beyond one $b_{o}$ occurs when the downwind vortex experiences a prolonged ground interaction.

- Results of all the simulations show that a layer-averaged ambient wind velocity can be used to bound the predicted time to travel across a specified distance, i.e. $t$ $=0.688 L \bar{U}$. This distance can represent the separation between parallel runways.
Future studies should address the sensitivity of wake transport to ambient wind shear both in and out of ground effect. Also. a select number of cases should be extended to three-dimensions to determine the effects of decay on wake transport.

\section{References}

[1] Hinton, D. A., 1995, "Aircraft Vortex Spacing System (AVOSS) Conceptual Design." NASA Tech. Memo No. 110184, 27 pp.

[2] Perry, R. B., D. A. Hinton, and R. A. Stuever, 1997, "NASA Wake Vortex Research for Aircraft Spacing," 35th Aerospace Sciences Meeting \& Exhibit, Reno. NV. AIAA 97-0057, 9 pp.

[3] Hinton, D. A., J. K. Charnock, D. R. Bagwell, and D. Grigsby, 1999, "NASA Aircraft Vortex Spacing System Development Status," 37th Aerospace Sciences Meeting \& Exhibit, Reno, NV, AIAA-99-0753, 17 pp.

[4] Schilling, V. K., 1992, "Motion and Decay of Trailing Vortices Within the Atmospheric Surface Layer," Beitr. Phys. Atmos., Vol. 65, pp. 157-169.

[5] Kopp. F., 1994, "Doppler Lidar Investigation of Wake Vortex Transport Between Closely Space parallel Runways," AIAA Journal. Vol. 32, pp. 805-810.

[6] Rudis. R. P., D. C. Burnham, and P. Janota, 1996, "Wake Vortex Decay Near the Ground under Conditions of Strong Stratification and Wind Shear," AGARD Fluid Dynamics Panel Symposium, Trondheim, Norway, AGARD CP-584, pp. 11-1 to 11-10.

[7] Robins, R. E. and D. P. Delisi, 1993, "Potential Hazard of Aircraft Wake Vortices in Ground Effect with Crosswind," J. Aircraft. Vol. 30, No. 2, pp. 20l-206.

[8] Critchley, J., and P. Foot, 1991, "UK CAA Wake Vortex Database: Analysis of Incidents Reported Between 1982 and 1990," Civil Aviation Authority, CAA Paper 91.

[9] Harvey, J. K., and F. J. Perry, 1971, "Flow field Produced by Trailing Vortices in the Vicinity of the Ground," AIAA Journal, Vol. 9, No. 8, pp. 1659-1660

[10] Hallock, J. N., 1975, "Wake Vortex Decay Near the Ground," AIAA 8th Fluid and Plasma Dynamics Conf.. Hartford, CN, AIAA-75-882, 7 pp.

[11] Hallock, J. N., and D. C. Burnham, 1997, "Decay Characteristics of Wake Vortices from Jet Transport Aircraft," 35th Aerospace Sciences Meeting \& Exhibit, AIAA-97-0060, 11 pp. 
[12] Burnham, D. C.. and J. N. Hallock, 1998. "Measurements of Wake Vortices Interacting with the Ground," 36th Aerospace Sciences Meeting \& Exhibit. AIAA-98-0593, 12 pp.

[13] Zheng, Z. C., and R. L. Ash, 1996, "Study of Aircraft Wake Vortex Behavior Near the Ground," AIAA Journal, Vol. 34, No. 3, pp. 580-589.

[14] Corjon, A., and T. Poinsot, 1997, "Behavior of Wake Vortices Near Ground," AIAA Journal, Vol. 35 , No. 5, pp. 849-855.

[15] Corjon, A., and A. Stoessel, 1997, "ThreeDimensional Instability of Wake Vortices Near the Ground," 28th AIAA Fluid Dynamics Conf., Snowmass, CO, AIAA-97-1782, 14 pp.

[16] Proctor, F. H., and J. Han, 1999, "Numerical Study of Wake Vortex Interaction with the Ground Using the Terminal Area Simulation System," 37th Aerospace Sciences Meeting \& Exhibit. Reno, NV, AIAA-99-0754, $12 \mathrm{pp}$.

[17] Proctor, F. H., D. W. Hamilton, and J. Han. 2000, "Wake Vortex Transport and Decay in Ground Effect: Vortex Linking with the Ground," 38th Aerospace Sciences Meeting \& Exhibit, Reno, NV, AIAA Paper No. 2000-0757, $14 \mathrm{pp}$.

[18] Burnham, D. C., 1972, "Effect of Ground Wind Shear on Aircraft Trailing Vortices," AIAA Journal, Vol. 10. No. 8, pp. 1114-1115.

[19] Proctor, F. H., 1998, "The NASA-Langley Wake Vortex Modeling Effort in Support of an Operational Aircraft Spacing System," 36th Aerospace Sciences Meeting \& Exhibit, Reno, NV, AIAA-98-0589, 19 pp.

[20] Zheng, Z. C., and K. Baek, 1998, "Inviscid Interactions Between Wake Vortices and Shear Layers." J. Aircruft, Vol. 36, No. 2, pp. 477-480.

[21] Sarpkaya, T., 2000, "A new model for vortex decay in the atmosphere," J. Aircruft, Vol. 37, pp. 53-61.

[22 ] Han, J., Y.-L. Lin, J. Zhang, S. P. Arya, "Large Eddy Simulation of Wake Vortices in the Convective Boundary Layer," Submitted to AlAA Journal. 25 pp.

[23] Proctor, F. H., 1987, "The Terminal Area Simulation System, Volume 1: Theoretical Formulation," NASA Contractor Report 4046, DOT/FAA/PM-85/50, 1 , $176 \mathrm{pp}$.

[24] Proctor, F. H., 1996, "Numerical Simulation of Wake Vortices During the Idaho Falls and Memphis Field Programs," 14th AIAA Applied Aerodynamics Conference, Proceedings, Part-II, New Orleans, LA, AIAA-96-2496, pp. 943-960.
[25] Switzer, G. F., 1996, "Validation Tests of TASS for Application to 3-D Vortex Simulations," NASA Contractor Report No. 4756, $11 \mathrm{pp}$.

[26] Han, J., Y.-L. Lin, D. G. Schowalter, S. P. Arya, and F. H. Proctor, 2000, "Large Eddy Simulation of Aircraft Wake Vortices within Homogeneous Turbulence: Crow Instability," AIAA Journal, Vol. 38, No. 2, pp. 292-300.

[27] Han, J., Y.-L. Lin, S. P. Arya, and F. H. Proctor, 2000, "Numerical Study of Wake Vortex Decay and Descent in Homogeneous Atmospheric Turbulence," AIAA Journal, Vol. 38, No. 4, pp. 643-656.

[28] Switzer, G. F. and F. H. Proctor, 2000, "Numerical Study of Wake Vortex Behavior in Turbulent Domains with Ambient Stratification," 38th Aerospace Sciences Meeting \& Exhibit, Reno, NV, AIAA Paper No. 2000$0755,14 \mathrm{pp}$.

[29] Shen, S., F. Ding. J. Han, Y-L. Lin, S. P. Arya, and F. H. Proctor, 1999, "Numerical Modeling Studies of Wake Vortices: Real Case Simulations," 37th Aerospace Sciences Meeting \& Exhibit, AIAA-99-0755, Reno, NV, $16 \mathrm{pp}$.

[30] Proctor, F. H., D. A. Hinton, J. Han, D. G. Schowalter, and Y.-L. Lin, 1997, "Two-Dimensional Wake Vortex Simulations in the Atmosphere: Preliminary Sensitivity Studies," 35th Aerospace Sciences Meeting \& Exhibit. Reno, NV, AIAA Paper No. 97-0056, $13 \mathrm{pp}$.

[31] Crow, S.C., 1970, "Stability Theory for a Pair of Trailing Vortices," AIAA Journal, Vol. 8, No. 12, pp. 2172-2179.

[32] Robins, R. E., D. P. Delisi, and G. C. Greene, 1998, "Development and Validation of a Wake Vortex Prediction Algorithm," 36th Aerospace Sciences Meeting \& Exhibit, AIAA-98-0665, 10 pp.

[33] Sarpkaya, T., 1983, "Trailing Vortices in Homogeneous and Density-stratified Media," Journal of Fluid Mech., Vol, pp. 85-109.

[34] Wickens. R. H., 1980, "A Technique for Simulating the Motion and Ground Effect of Aircraft Wake Vortices," Canadian Aeronautics and Space Journal, Vol. 26, No. 2, pp. 129-133.

[35] Heinrichs, R. M., T. J. Dasey, M. P. Matthews, S. D. Campbell, R. E. Freehart, G. H. Perras, and P. Salamitou, 1996, "Measurements of Aircraft Wake Vortices at Memphis International Airport with a CW CO2 Coherent Laser Radar," SPIE Symposium on Aerospace/Defense Sensing and Controls, Air Traftic Control Technologies II, SPIE-2737-20, Orlando, FL, 11 pp. 